\title{
Optimal channel design and sensor placement in flow distributors for detecting blockage of parallelized microreactors
}

\section{$\operatorname{AUTHOR}(S)$ :}

Tonomura, Osamu; Nishida, Atsushi; Wang, Lin; Hasebe, Shnji

\section{CITATION:}

Tonomura, Osamu ... [et al]. Optimal channel design and sensor placement in flow distributors for detecting blockage of parallelized microreactors. Computer Aided Chemical Engineering 2012, 31: 1281-1285

\section{ISSUE DATE:}

2012

URL:

http://hdl.handle.net/2433/160125

\section{RIGHT:}

C 2012 Elsevier B.V.; この論文は出版社版でありません。引用の際には 出版社版をご確認ご利用ください。; This is not the published version. Please cite only the published version. 


\title{
Optimal channel design and sensor placement in flow distributors for detecting blockage of parallelized microreactors
}

\author{
Osamu Tonomura, Atsushi Nishida, Lin Wang, Shnji Hasebe \\ Dept. of Chemical Engineering, Kyoto University, Nishikyo, Kyoto 615-8510, Japan. \\ E-mail: tonomura@cheme.kyoto-u.ac.jp (O. Tonomura)
}

\begin{abstract}
When the production capacity of micro chemical plants is increased by numbering-up approach, it is important to realize the uniform flow distribution among the parallelized microreactors. In addition, a blocked microreactor needs to be identified as early as possible to achieve the stable long-term operation of micro chemical plants. In this research, a system that can detect a blocked microreactor by using just two flow sensors is developed. The performance of the developed system is maximized by adjusting the channel size and sensor placement in the flow distributors. The effectiveness of the developed system and the optimal design result is demonstrated by computational fluid dynamics simulation.
\end{abstract}

Keywords: microreactor, flow distributor, design, sensor placement, blockage detection.

\section{Introduction}

Microreactors engage the attention of researchers and engineers in the pharmaceutical and chemical industries. Microreactors can achieve the high yield and selectivity of a product owing to their advantages of rapid mixing, high heat transfer, and precise residence time control (Hessel et al., 2005). The numbering-up approach, which means the parallelization of microreactors, is effective at increasing the production capacity of microreactors and opens up the possibility of a direct transfer from laboratory scale to production scale. However, some difficulties are left behind. When the numbering-up approach is applied to micro chemical plants (MCPs), it is important to realize the uniform flow distribution among the parallelized microreactors, which are connected by flow distributors. The flow maldistribution is caused by the inadequate design of the flow distributors and deteriorates the performance of MCPs. In addition to the flow uniformity, it is necessary to take measures against blockage, which is the most recognized trouble with MCPs. For example, Bayer et al. (1999), who developed a continuous radical polymerization process having micro pre-mixers, mentioned that poor mixing conditions cause blockage in a reactor. Ju et al. (2006) applied a stainless steel microchannel reactor for the continuous synthesis of zeolite $\mathrm{NaA}$, and they found that aging the synthesis solution was a key procedure for avoiding blockage of the microchannel. In these literatures and others, one of the critical operation problems was blockage in microreactors, which negatively affects the flow distribution in the whole MCPs with numbering-up structure. Therefore, a blocked microreactor needs to be detected as early as possible to achieve the stable long-term operation of MCPs. However, it is not practical to install the sensors in all the microreactors from the viewpoint of cost or space. Therefore, the goals of this research are to develop a flow 
distributor suitable for MCPs and to propose a blockage detection method with the limited measurement.

\section{Flow Distribution in Parallelized Microreactors}

This section shows the basic structure and characteristics of MCPs with parallelized microreactors. The characteristics of a specific flow distributor developed by Tonomura et al. (2008) are also analyzed through computational fluid dynamics (CFD) simulations.

2.1. Split-and-recombine-type flow distributors (SRFDs)

A schematic diagram of a MCP with externally parallelized microreactors is shown in Fig. 1. The parallelized section where microreactors are running in parallel is connected to a distribution section and a confluence section. The distribution section carries out a function of distributing a reactant flow among the parallelized microreactors. The distributed flows pass through microreactors and are collected into one flow at the confluence section. A lack of uniformity in the flow distribution lowers the MCP's performance.

Flow distributors, which are used at the distribution section in Fig. 1, are typically classified into two types: manifold-type and bifurcation-type. Unlike such flow distributors, Tonomura et al. (2008) have developed split-and-recombine-type flow distributors (SRFDs). Figure 2 shows an example of SRFDs, in which one flow is divided into four flows. The SRFD consists of channels having a length of $L[\mathrm{~mm}]$ and a width of $d[\mathrm{~mm}]$. It is assumed that $L_{1}=L_{4}=L$ and $L_{2}=L_{3}=\alpha L . \alpha$ is introduced to achieve the uniform flow distribution among the parallelized microreactors under a normal operating condition and determined by CFD simulation. $u_{\text {in }}[\mathrm{m} / \mathrm{s}]$ is the averaged flow velocity at the inlet, and $u_{\mathrm{k}}[\mathrm{m} / \mathrm{s}]$ ( $k$ is channel number) is the averaged flow velocity at the outlet of each channel. The outlet of SRFD is opened to atmosphere. In this research, the commercial CFD software "COMSOL Multiphysics ${ }^{\circledR}$ 3.4" is used to estimate the flow distribution of water (293 K) in the SRFD. The normalized flow velocity $u^{*}{ }_{k}$, which is given by $u_{k} / u_{\text {in }}$, is used to evaluate the flow distribution.



Figure 1 A MCP with parallelized microreactors.



Figure 2 A developed SRFD.

\subsection{Design modification of SRFD}

It is preferable that the uniform flow distribution in the SRFD is maintained even when the inlet flow velocity or the material properties of the fluid change. The effects of 
design parameters on the flow distribution are investigated by using CFD simulation. Table 1 shows the representative simulation conditions for SRFDs. When a circular channel having $L=10 \mathrm{~mm}$ and $d=1.0 \mathrm{~mm}$ is adopted, $u_{\text {in }}=0.2 \mathrm{~m} / \mathrm{s}$ corresponds to inlet volumetric flowrate $V_{\text {in }}=10 \mathrm{~mL} / \mathrm{min}$. Figure 3 shows the results of CFD simulation. The vertical axis denotes the differences between two normalized flow velocities, $u^{*}$ and $u_{1}^{*}$, and the flow distribution is highly uniform when each plotted point in Fig. 3 is located near zero. The uniform flow distribution is achieved independently of $L / d$, when Reynold number $(\mathrm{Re})$ is less than 100 . At higher Re, the difference in flow velocity between Ch. 2 and Ch. 1 becomes large. In such a case, the SRFD needs to be redesigned so as to realize the uniform flow distribution. The alternative approach is to use flow controllers. However, the installation of flow controllers in all the channels requires high cost and extra space.

Figure 3 indicates that the difference between $u_{2}^{*}$ and $u_{1}^{*}$ is reduced at higher Re, according as $L / d$ becomes large. An increase in $L / d$ usually leads to a larger pressure drop of flow in the SRFD $\left(\Delta P_{\mathrm{SRFD}}\right)$. To further improve the uniformity in the flow velocities without increasing $\Delta P_{\mathrm{SRFD}}$, the modified SRFD (mSRFD) where the channel width of Ch. 0 is enlarged, as shown in Fig. 4, is proposed in this research. CFD simulation result of mSRFD $(L / d=20)$ is added to Fig. 3 , and it is clarified that mSRFD gives a more uniform flow distribution in the wide range of Re.

Table 1 Representative simulation conditions for SRFDs.

\begin{tabular}{rlllll}
\hline$L / d$ & $L[\mathrm{~mm}]$ & $d[\mathrm{~mm}]$ & $\alpha$ & $u_{\text {in }}[\mathrm{m} / \mathrm{s}]$ & $\operatorname{Re}$ \\
\hline 5 & 10 & 2.0 & 1.80 & 0.05 & 100 \\
10 & 10 & 1.0 & 1.75 & 0.2 & 200 \\
20 & 10 & 0.5 & 1.78 & 0.8 & 400 \\
40 & 40 & 1.0 & 1.67 & 0.2 & 200 \\
\hline
\end{tabular}

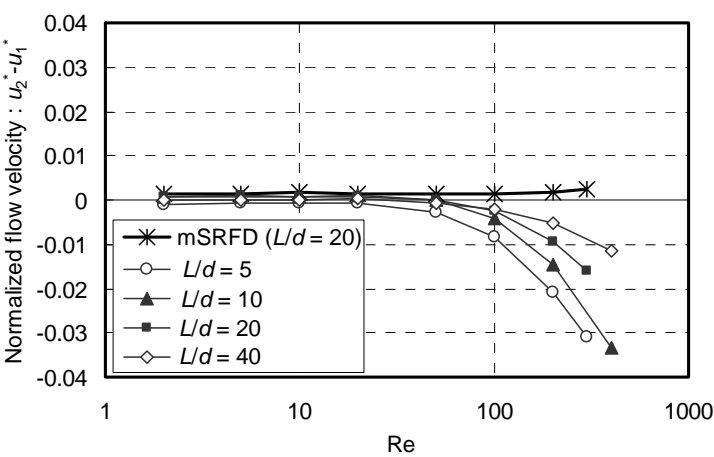

Figure 3 CFD simulation results.

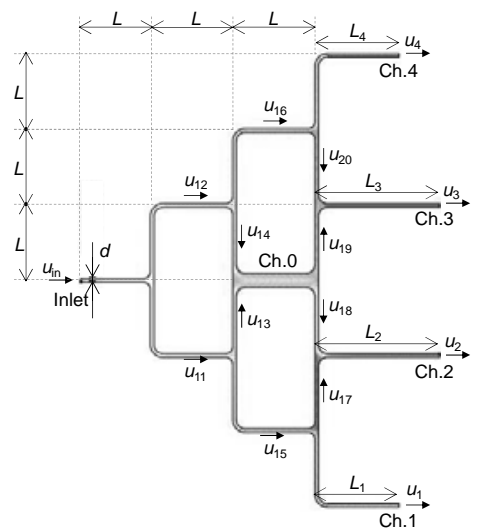

Figure 4 A modified SRFD.

\section{Blockage Detection in Parallelized Microreactors}

Blockage is the most recognized problem in MCPs, because the diameter of microchannels is less than $1 \mathrm{~mm}$. A process monitoring system that can detect blockage is indispensable for effective and stable operation of MCPs. In this research, just two flow sensors are embedded in SRFDs, and a blockage diagnosis system (BDS) is 
developed to identify a blocked microreactor among the parallelized microreactors. In addition, the performance of the developed system is maximized by adjusting the channel size and sensor placement in the flow distributors.

\subsection{Blockage diagnosis system}

Reactants are supplied to MCPs at a constant flowrate. MCPs have a symmetric structure and are designed beforehand so that flow distribution is uniform. Under these assumptions, the developed BDS uses the ratios of flow velocity (or flowrate) differences between normal and abnormal operating conditions at one sensor to those at the other sensor. In this research, two sensors are located in SRFDs. The procedure for building BDS is as follows: (1) obtain flow velocity data under normal operating conditions, (2) obtain flow velocity data under blockage in each microreactor, (3) calculate the flow velocity difference between normal and abnormal operating conditions at two sensors, and (4) calculate the ratio of flow velocity differences. After blockage is detected, the blockage location is identified through the following procedure: (1) calculate the flow velocity difference between normal and abnormal operating conditions at two sensors, (2) calculate the ratio of flow velocity differences, and (3) identify the microreactor that has the smallest difference between the prepared ratio and the actual ratio as a blocked reactor.

\subsection{Application to four parallelized microreactors}

The performance of the developed BDS is evaluated with its application to four parallelized microreactors, which are connected to SRFD. Through the system development, the results of flow velocity measurements are plotted in the two dimensional $\Delta u_{15}$ and $\Delta u_{16}$ space (Fig. 5). In this figure, $\Delta P_{\mathrm{MR}}$ and $\Delta P_{\mathrm{mSRFD}}$ represents the pressure drop of microreactors and $\mathrm{mSRFD}$, respectively. The ratios of flow velocity differences under the blockage in microreactor connected to Ch. $i$ ( $i=1,2,3,4)$ correspond to square, diamond, triangle, and circle, respectively. As each point deviates from the origin, the total flowrate in the process becomes large. Figure 5 shows that the

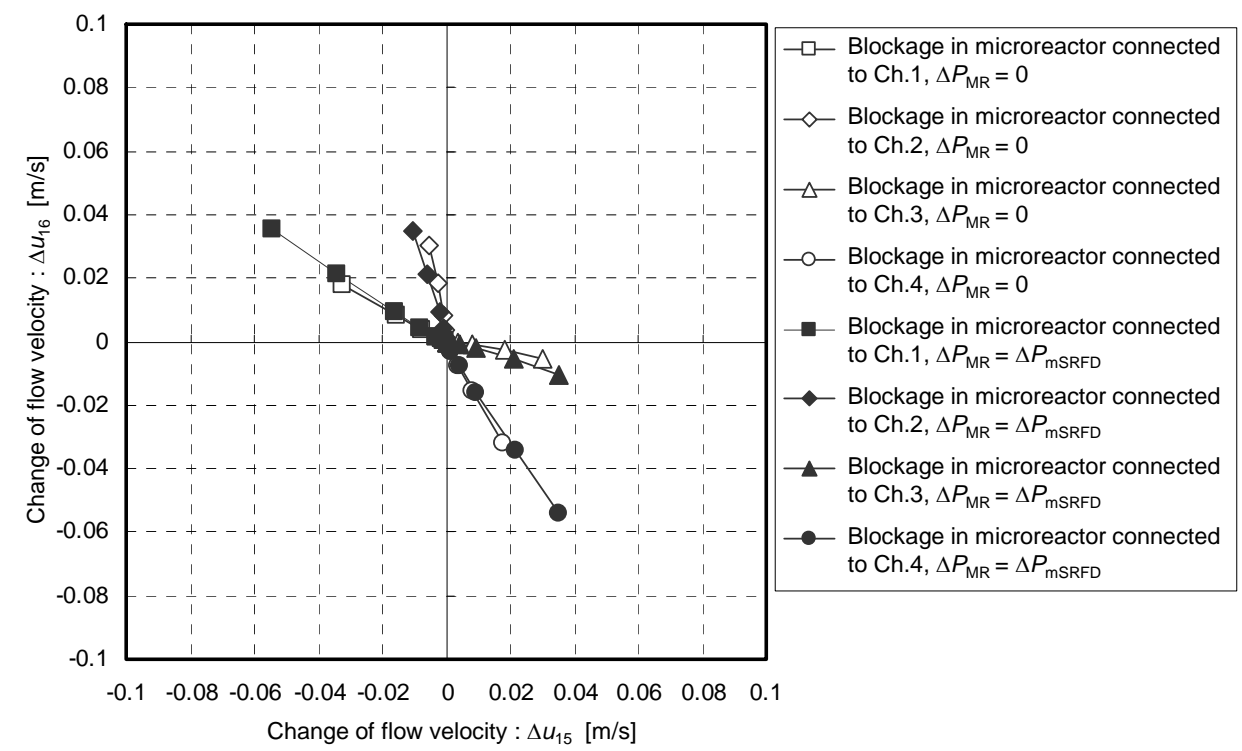

Figure 5 Blockage diagnosis results (Flow sensors are located at $u_{15}$ and $u_{16}$ ). 
ratio of two flow velocity differences remains unchanged even when total flow rate of the process varies, without reference to $\Delta P_{\mathrm{MR}}$. In addition, it is clarified that the blockage location is successfully identified on the basis of the ratios of two flow velocity differences. These simulation results suggest that the developed BDS has robustness to changes in the total flowrate and $\Delta P_{\mathrm{MR}}$.

\subsection{Optimization of channel size and sensor placement}

Flow distribution in MCPs with/without blockage can be estimated by CFD model or pressure balance (PB) model (Amador et al., 2004). In this research, an optimal channel design and sensor placement problem is solved on the basis of PB model, which requires less computational time than CFD model. Channel diameters and sensor placement are determined to maximize the adjacent angles between lines in Fig. 5 within constraints on the total flowrate, the flow uniformity under normal operating condition, the number of available sensors, the number of parallelized microreactors and their pressure drop, and the channel size boudaries. As a result of optimization by using gPROMS ${ }^{\circledR}$, the maximum angle is obtained when the diameters of horizontal and vertical channels of the SRFD are $2.8 \mathrm{~mm}$ and $2.1 \mathrm{~mm}$, respectively, and the two sensors are located at channels of $u_{17}$ and $u_{20}$. The effectiveness of the optimal design result is confirmed by CFD simulation.

\section{Conclusions}

The flow uniformity in SRFDs, which have three or more bifurcation points and one or more junction points, was examined through CFD simulation, and the design of SRFDs is modified to achieve a uniform flow distribution in the wide range of Re. In addition, the blockage detection and diagnosis system that can identify a blocked microreactor by using a small number of flow sensors was developed, and its effectiveness was demonstrated numerically. The results showed that the developed system has high robustness to changes in the fluid properties and the microreactor characteristics such as pressure drop. In addition, the performance of the developed system was maximized by adjusting the channel size and sensor placement in the flow distributors. The developed system will be applicable to various types of microchemical plants with parallelized microreactors.

\section{References}

C. Amador, A. Gavriilidis, and P. Angeli, 2004, Chem. Eng. J., 101, 379-390.

T. Bayer, D. Pysall, and O. Wachsen, 1999, Proc. of the $3^{\text {rd }}$ International Conference on Microreaction Technology (IMRET3), Frankfurt, Germany, 165-170.

V. Hessel, S. Hardt, and H. Loewe, 2005, Chemical Micro Process Engineering: Fundamentals, Modelling and Reactions, Wiley, VCH, Weinheim.

J. Ju, C. Zeng, L. Zhang, and N. Xu, 2006, Chem. Eng. J. 116, 115-121.

O. Tonomura, S. Nagahara, M. Kano, and S. Hasebe, 2008, Proc. of $10^{\text {th }}$ International Conference on Microreaction Technology/AIChE Spring National Meeting, New Orleans, US, 128d. 